\title{
Prozessoptimierung durch ganzheitliches Bettenmanagement im Krankenhaus
}

Das Krankenhausbett ist Dreh- und Angelpunkt der stationären Leistungserbringung. Fehllagerungen, Leerzeiten, ineffizientes Reinigungs- und Instandhaltungsmanagement sowie vermeidbarer Überbestand führen zur Bindung wertvoller Ressourcen.

Das Bettenmanagement gestaltet sich in Deutschland vielfältig. Prozesse, wie die Beschaffung, Aufbereitung, Instandhaltung, Lagerung oder Entsorgung kommen in der deutschen Kliniklandschaft in den verschiedensten Umsetzungsvarianten vor. Betrachtet man die Bettenaufbereitung, so ergeben sich verschiedenste Ausprägungen der Organisation, Koordination und Durchführung (Abbildung 1).

Die Relevanz des Bettenmanagements wird im OECD-Ländervergleich deutlich. So weist Deutschland die höchste Krankenhausentlassungsrate auf, welche mit hohen Anforderungen an Aufbereitung und Instandhaltung von Krankenhausbetten verbunden ist. ${ }^{1}$ Organisation und Workflow richten sich nach den lokalen Voraussetzungen und unterliegen der Entscheidung des Trägers, des Managements sowie der Ausführenden. Faktoren wie Bettenbestand, Baukörper und Transporttechnik nehmen zusätzlich entscheidenden Einfluss.

Das Bettenmanagement gewinnt im Zuge steigender Fallzahlen und sinkender Verweildauern (Abbildung 2) weiter an Bedeutung, da sich hieraus eine entsprechend erhöhte Umschlaghäufigkeit der Krankenhausbetten ergibt.

Dieser Trend wird sich laut Experten in den nächsten Jahren weiter fortsetzen; so wird gemäß RWI - Leibniz-Institut für Wirtschaftsforschung mit einer durchschnittlichen Verweildauer (VWD) von 6,2 Tagen bzw. einer Steigerung der Fallzahlen um 6 Prozent im Jahre 2030 gerechnet. Die

Wie erfolgt die Bettenaufbereitung? Ergebnisse einer Kurzbefragung zur Bettenaufbereitung

Abb. 1, (Krankenhäuser in Prozent)

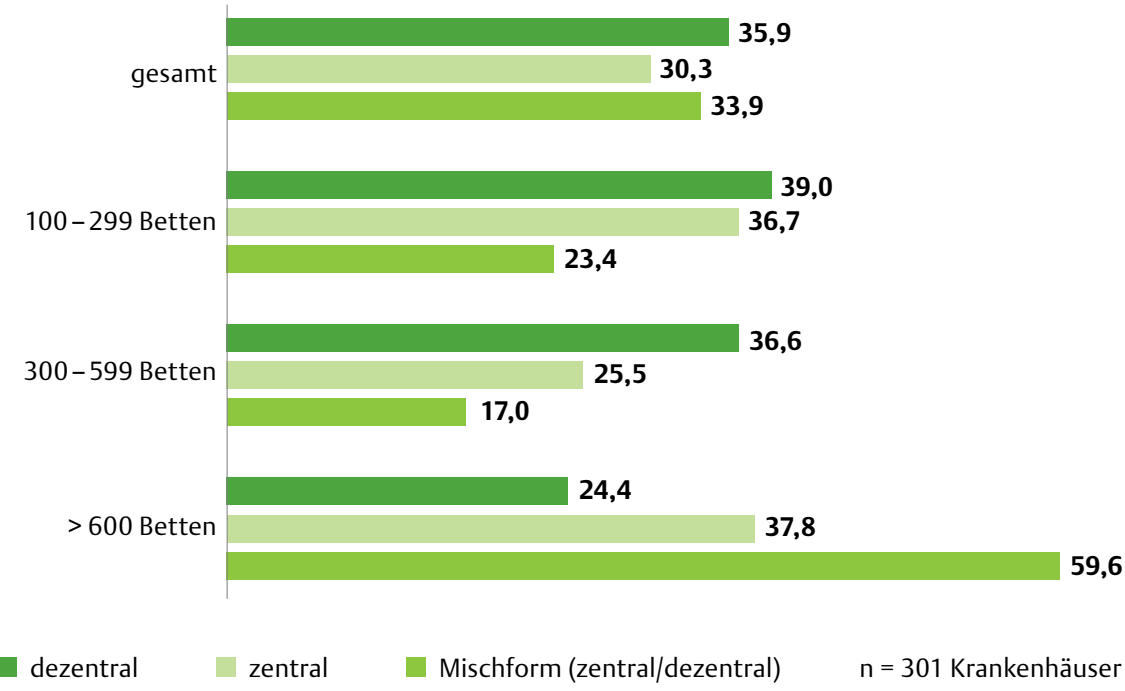

Quelle: Dr. Matthias Offermanns (DKI)

damit verbundene Bereitstellung und Instandhaltung von Krankenhausbetten verlangen daher eine nachhaltige Effizienzsteigerung des Bettenmanagements. Thomas Weyers (Pflegedirektor der St. Josef Krankenhaus GmbH Moers) kann das nur bestätigen und weist darauf hin, dass auch ,heute schon die zeitgerechte Aufbereitung eines hygienisch einwandfreien Bettes im Rahmen des Belegungsmanagements elementarer Bestandteil der Versorgungsstruktur ist“.

Das Projekt Bettenmanagement 4.0 Im Rahmen des, aus Mitteln des Europäischen Fonds für regionale Entwicklung (EFRE), geförderten Projektes „Bettenmanagement 4.0: Mehr Sicherheit und
Ressourcen durch Bettenmanagement 4.0 im Krankenhaus“ wird eine ganzheitliche, lebenszyklus- und bedarfsorientierte Prozessoptimierung im Krankenhaus angestrebt. Damit sollen bestehende Ressourcen geschont, die Sicherheit für Krankenhausmitarbeiter und Patienten erhöht sowie die Logistik im stationären Gesundheitssektor gestärkt werden. Konkret erfolgt die Umsetzung durch eine bedarfsgerechte und herstellerunabhängige IT-Systemlösung.

Als Anwender stehen die beiden Krankenhäuser St. Marien-Hospital Mülheim an der Ruhr und St. Josef Krankenhaus Moers als assoziierte Partner zur Verfügung. Dort sollen die Lösungen implementiert und 


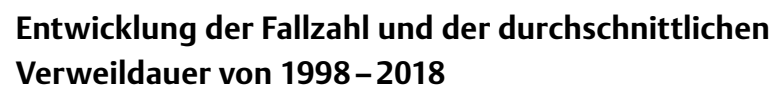

Abb. 2
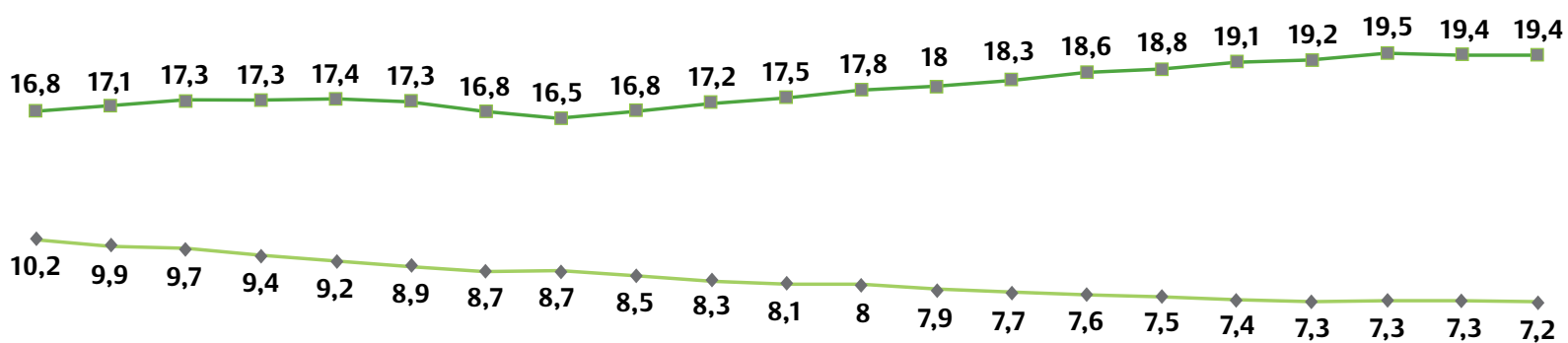

getestet werden. Die Konsortialführung übernimmt die medmehr $\mathrm{GmbH}$, die Services für das Gesundheitswesen anbietet. Dr.-Ing. Mandana Banedj-Schafii erklärt ihre Motivation, das Projekt ins Leben gerufen zu haben damit, dass „medmehr als technischer Dienstleister in langjähriger Erfahrung angesichts der aktuellen Situation in vielen Krankenhäusern einen hohen Bedarf für ein leicht umsetzbares, funktionierendes Schnittstellenmanagement rund um das Krankenhausbett sieht“.

Zu den Verbundpartnern zählen die Hochschule Niederrhein, die Fachhochschule Dortmund, das Deutsche Krankenhausinstitut (DKI) und die Ruhr-Universität Bochum. Assoziierte Partner sind neben den genannten Krankenhäusern die beiden Fachverbände „Wissenschaftliche Gesellschaft für Krankenhaustechnik“ (WGKT) und die „Fachvereinigung Krankenhaustechnik“ (FKT).

\section{Bedarfsanalyse}

Im Zuge strukturierter Ist-Erhebungen mehrerer stationärer Gesundheitseinrichtungen wurden erhebliche Verzögerungen klinischer Prozessabläufe erkannt, die u.a. auf eine uneinheitliche (d.h. auch hausintern unterschiedliche)
Prozessgestaltung der Bettenaufbereitung zurückgeführt werden konnten. Eine quantitative Bewertung dieser Situation bzw. der damit verbundenen Missstände ist nicht vollständig abbildbar, weil die hierzu erforderlichen Zeitstempel häufig fehlen. Derartige Probleme konnten bereits in der Vergangenheit durch Untersuchungen der Hochschule Niederrhein aufgezeigt werden, bei denen selbst Krankenhäuser mit einem Logistikleitstand eine zum Teil hohe Zahl von fehlerhaften Zeitstempeln im Bettentransport aufwiesen.

Für ein funktionierendes, an den Bedarf angepasstes Bettenmanagement fehlt im Weiteren meist,

- die aktuelle Lokalisation aller (verfügbaren) Betten,

- der aktuelle Zustand/Status, wie z.B. belegbar, verschmutzt, in Instandhaltung,

- Echtzeit-Kommunikation über die Entlassung und Koordination der Bettenaufbereitung.

Weiterhin ist festzustellen, dass sowohl Entscheidungsträger als auch operatives Personal in vielen Fällen keine Übersicht über den aktuellen Bettenbestand haben. Dies gilt sowohl in quantitativer (Anzahl
Die Hauptfunktionen des Systems:

$\checkmark$ raumgenaue Lokalisierung,

- Informationen über den aktuellen Bettenzustand,

- Anforderung der Bettenaufbereitung in Echtzeit nach Patientenentlassung,

- Übersicht des Instandhaltungsbedarfs sowie die Möglichkeit der dynamischen Instandhaltungssteuerung auf Grundlage der Belegungssituation,

- Schnittstellen zu vorhandenen CAFM-Systemen (ComputerAided Facility Management) sowie Dokumentenmanagementsystemen zur Nachverfolgung von medizintechnischen Dokumenten und Einweisungsmaterial für Schulungen,

> strukturierte Bereitstellung von Daten zur Unterstützung operativer und strategischer Entscheidungen im Lifecycle (d.h. von der Beschaffung bis hin zur Verwertung/Entsorgung). 


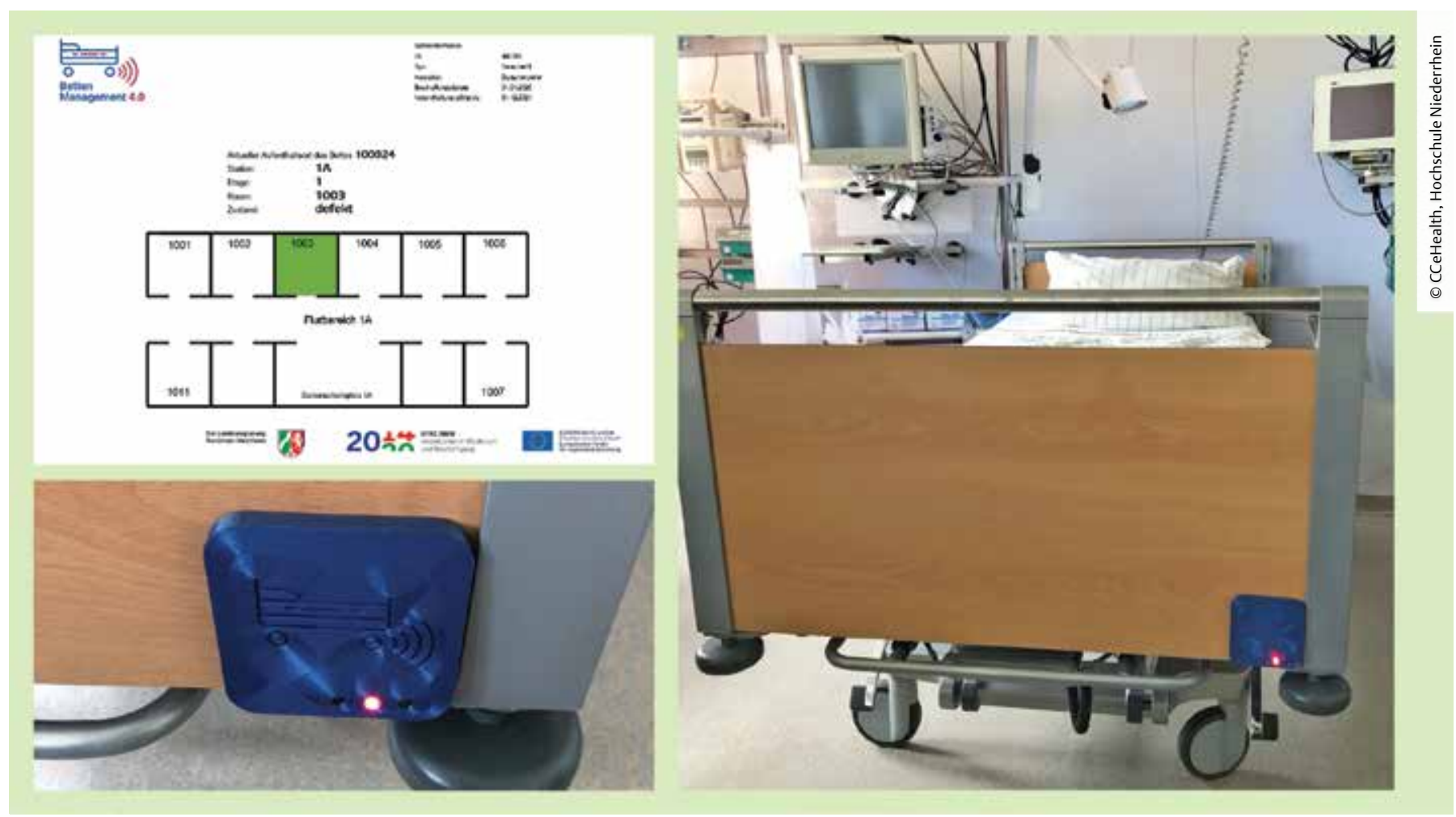

Konzeptgrafik der ITSystem-Lokalisierung (c) CCeHealth, Hochschule

verschiedener Typen und Modelle, Bettenüberhang, Reparaturhäufigkeit, LifetimeKosten usw.) als auch qualitativer (z.B. Instandhaltungszustand) Hinsicht.

\section{Lösungsvorschlag}

Aufbauend auf den Ist-Erhebungen, der damit einhergehenden Schwachstellenanalyse sowie dem Fachwissen des Projektkonsortiums und weiterer Experten aus den Bereichen der Medizintechnik, Krankenhauslogistik, Prozessmanagement und Pflege, erfolgte die Ableitung einrichtungsübergreifender Soll-Prozesse, einschließlich notwendiger Datenquellen und Datenpunkte (u.a. Zeitstempel, Lagerort, Bettenzustand etc.), technischer Unterstützungssysteme und Prozessschnittstellen, die neben dem Workflow auch angebundene IT-Systeme berücksichtigen. Darüber hinaus stellen die Grundanforderungen der Nutzer einen Schwerpunkt dar, um sowohl eine effiziente Prozessintegration sicherzustellen als auch die Entwicklung des IT-Systems, bestehend aus Hard- und Softwarekomponenten, zu komplementieren.

\section{IT-System}

Das zur Anwendung kommende IT-System soll die Prozesse so unterstützen, dass die relevanten Nutzergruppen durch die geplanten Funktionen bedarfsgerecht unterstützt werden. Einen hohen Stellenwert nimmt dabei die Akzeptanz der zukünftigen Nutzer ein. Betrachtet man die aktuelle Arbeitsbelastung derselben, so ist zwingend jede Form einer Zusatzbelastung, speziell im Bereich der Gesundheits- und Krankenpflege zu vermeiden. Hierzu werden durch das Projektkonsortium verschiedenste Möglichkeiten der Mensch-Computer-Interaktion getestet und evaluiert. Die entwickelte Hardware wird dazu in geeigneter Form mit dem Bett verbunden. Die Integration bzw. Anmeldung eines Bettes ins System erfolgt per Plug-and-play und ist damit auch für Mitarbeiter aus dem Service-Bereich ohne fundierte IT-Kenntnisse unproblematisch durchführbar.

\section{Ausblick}

In der Hoffnung auf eine Entspannung der Corona-Pandemie sind erste Testläufe im klinischen Umfeld zum Ende des Jahres 2021 geplant. Die anhand der Tests gewonnenen Erkenntnisse sollen im weiteren Verlauf in die Verbesserung nicht nur des IT-Systems, sondern insbesondere der Soll-Prozesse, einfließen. Hierbei wird der Fokus auf die Validität der verfügbaren
Daten sowie auf die Integration weiterer Datenquellen gelegt. Ein wichtiger Aspekt stellt ferner die bedarfsgerechte Anpassung und Optimierung der verschiedenen Benutzerschnittstellen dar. Eine zum Ende der Pilotphase vorgesehene Nutzenbewertung neu gestalteter Prozesse sowie des IT-Systems selbst soll neben qualitativen und quantitativen Verbesserungen insbesondere auch die Entlastung in Medizin und Pflege aufzeigen.

\section{- Quellen}

1 OECD (2021), Hospital discharge rates (indicator). doi: 10.1787/5880c955-en (Accessed on 26 April 2021)

Prof. Dr.-Ing. Hubert Otten ist Leiter des Instituts für Health Care Management und des Competence Center eHealth (CCeHealth) an der Hochschule Niederrhein.

Benjamin Jagusch, B.Sc. leitet das Projekt Bettenmanagement $4.0 \mathrm{im}$ cCeHealth.

Nico Schnee, B.Sc. ist wissenschaftlicher Mitarbeiter im CCeHealth. 